\title{
Morphology and deep metazoan phylogeny
}

\author{
Andreas Schmidt-Rhaesa
}

Published online: 24 June 2009

(C) Springer-Verlag 2009

The broad attempts to reconstruct phylogenetic relationships of organisms are reflected in several small and large projects in many different countries. In Germany, the focal priority program 1174 of the German Science Council (Deutsche Forschungsgemeinschaft, DFG) called Deep Metazoan Phylogeny aims to add new data to phylogenetic analyses. The program unites molecular and morphological approaches; this Special Volume will present process of the morphological projects. Please note that the three articles by Harzsch et al. (2009), Stach (2009) and Suschenko and Purschke (2009) also belong into this Special Volume, but were printed earlier due to restricted space.

The current power of morphological research for phylogenetic research has been doubted on different occasions. The argument that morphology has failed to resolve several phylogenetic relationships and should give way to molecular methods is more political than scientific. Now it is generally recognized that molecular methods suffer from the same amount and range of problems as morphological methods do. The value of morphology becomes more and more appreciated (see, e.g., Bartolomaeus 2003; Budd and Olsson 2007; Harrison 2007). In the context of phylogenetic research, morphology should be appreciated for (at least) two reasons.

First, molecular methods produce trees, but any explanatory power behind these trees is only possible in combination with morphological data. By combining a tree topology with morphological characters, evolutionary changes within the organisms themselves become evident,

\footnotetext{
A. Schmidt-Rhaesa $(\square)$

Zoological Museum and Biocenter Grindel,

University of Hamburg, Martin-Luther-King-Platz 3,

20146 Hamburg, Germany

e-mail: andreas.schmidt-rhaesa@uni-hamburg.de
}

and this is in the last sense what we want to find out. Therefore, we need detailed information on morphology to recognize such evolutionary changes.

Second, morphological research is not as old-fashioned as some critiques imply. There have been considerable developments during the past decades concerning microscopical investigation and reconstruction methods. The integration of histochemical (nuclear stainings, staining of f-actin with phalloidin) and immunohistochemical methods allowed the targeted search for the distribution of particular molecules within an organism. Methods such as confocal laser scanning microscopy produce 3D digital images and raise, together with adequate software, the visualization and documentation of structures on a new high quality level. Powerful programs and computers allow 3D-reconstructions form large stacks of micrographs obtained by light and electron microscopies in a way no one could imagine a decade ago. Finally, $\mu \mathrm{CT}$ and NMR techniques meanwhile lead to produce stacks of images that are already aligned and enable visualization of structures so far hardly visible due to invasive technologies (see Ziegler et al. 2008). Due to these non-invasive techniques we also have access to rare material from museums or type material.

Instead of persisting on the differences between morphological and molecular approaches to phylogeny, it must be stressed how intimately they are connected and how valuable they are for each other. Indeed, the gap between these two approaches is becoming smaller by such methods that visualize the distribution of single molecules or of gene expression patterns. Evolutionary developmental biology is sitting in this transition area.

The focal program "Deep Metazoan Phylogeny" includes both molecular and morphological projects for exactly these reasons: it is expected that both will produce new data of high quality and value for phylogenetic analyses 
and that both together are important for the entire picture of animal evolution.

The morphological projects within "Deep Metazoan Phylogeny" agreed to concentrate on three areas: nervous systems, cleavage and gastrulation as well as mesodermal structures, in particular body cavities. We start this Special Volume with a review on the need for a standardized language for morphological descriptions (Vogt 2009), because besides the search for new characters it is equally important to develop appropriate methods to deal with such data. Contributions of Harzsch et al. (2009), Rothe and SchmidtRhaesa (2009), Heuer and Loesel (2009) and Suschenko and Purschke (2009) present new data on the structure of the nervous system and photoreceptors. Scholtz and Ungerer investigate the cleavage and gastrulation in pycnogonids. Stach (2009) and Bartolomaeus et al. (2009) provide new insights into mesodermal structures.

\section{References}

Bartolomaeus T (2003) Change and continutiy. Zoomorphology 122:1-2

Bartolomaeus T, Quast B, Koch M (2009) Nephridial development and body cavity formation in Artemia salina (Crustacea: Brachiopoda): no evidence for any transitory coelom. Zoomorphology. doi:10.1007/s00435-008-0082-4
Budd GE, Olsson L (2007) Editorial: a renaissance for evolutionary morphology. Acta Zoologica 88:1

Harrison FW (2007) 1807-2007: a great anniversary celebration. J Morphol 268:103-111

Harzsch S, Müller CHG, Rieger V, Perez Y, Sintoni S, Sardet C, Hanson B (2009) Fine structure of the ventral nerve centre and intraspecific identification of individual neurons in the enigmatic Chaetognatha. Zoomorphology 128:53-73

Heuer CM, Loesel R (2009) Three-dimensional reconstruction of mushroom body neuropils in the polychaete species Nereis diversicolor and Harmothoe areolata (Phyllodocida, Annelida). Zoomorphology. doi:10.1007/s00435-008-0063-7

Rothe BH, Schmidt-Rhaesa A (2009) Architecture of the nervous system in two Dactylopodola species (Gastrotricha, Macrodasyida). Zoomorphology. doi:10.1007/s00435-008-0077-1

Scholtz G, Ungerer P (2009) Cleavage and gastrulation in Pycnogonum litorale (Arthropoda, Pycnogonida): morphological support for the Ecdysozoa? Zoomorphology. doi:10.1007/s00435-0090091-y

Stach TG (2009) Anatomy of the trunk mesoderm in tunicates: homology considerations and phylogenetic interpretation. Zoomorphology 128:97-109

Suschenko D, Purschke G (2009) Ultrastructure of pigmented adult eyes in errant polychaetes (Annelida): implications for annelid evolution. Zoomorphology 128:75-96

Vogt L (2009) The future role of bio-ontologies for developing a general data standard in biology: chance and challenge for zoo-morphology. Zoomorphology. doi:10.1007/s00435-008-0081-5

Ziegler A, Faber C, Mueller S, Bartolomaeus T (2008) Systematic comparison and reconstruction of sea urchin (Echinoidea) internal anatomy: a novel approach using magnetic resonance imaging. BMC Biol 6:33 